\title{
The Boundedness of Some Integral Operators on Weighted Hardy Spaces Associated with Schrödinger Operators
}

\author{
Hua Wang \\ College of Mathematics and Econometrics, Hunan University, Changsha 410082, China \\ Correspondence should be addressed to Hua Wang; wanghua@pku.edu.cn
}

Received 21 July 2015; Accepted 22 October 2015

Academic Editor: Dashan Fan

Copyright (C) 2015 Hua Wang. This is an open access article distributed under the Creative Commons Attribution License, which permits unrestricted use, distribution, and reproduction in any medium, provided the original work is properly cited.

Let $L=-\Delta+V$ be a Schrödinger operator acting on $L^{2}\left(\mathbb{R}^{n}\right), n \geq 1$, where $V \not \equiv 0$ is a nonnegative locally integrable function on $\mathbb{R}^{n}$. In this paper, we will first define molecules for weighted Hardy spaces $H_{L}^{p}(w)(0<p \leq 1)$ associated with $L$ and establish their molecular characterizations. Then, by using the atomic decomposition and molecular characterization of $H_{L}^{p}(w)$, we will show that the imaginary power $L^{i \gamma}$ is bounded on $H_{L}^{p}(w)$ for $n /(n+1)<p \leq 1$, and the fractional integral operator $L^{-\alpha / 2}$ is bounded from $H_{L}^{p}(w)$ to $H_{L}^{q}\left(w^{q / p}\right)$, where $0<\alpha<\min \{n / 2,1\}, n /(n+1)<p \leq n /(n+\alpha)$, and $1 / q=1 / p-\alpha / n$.

\section{Introduction}

Let $n \geq 1$ and $V$ be a nonnegative locally integrable function defined on $\mathbb{R}^{n}$, not identically zero. We define the form $\mathbb{Q}$ by

$$
\mathbb{Q}(u, v):=\int_{\mathbb{R}^{n}} \nabla u \cdot \nabla v d x+\int_{\mathbb{R}^{n}} V u v d x
$$

with domain $\mathscr{D}(\mathscr{Q})=\mathscr{V} \times \mathscr{V}$, where

$$
\begin{aligned}
\mathscr{V} & :=\left\{u \in L^{2}\left(\mathbb{R}^{n}\right): \frac{\partial u}{\partial x_{k}} \in L^{2}\left(\mathbb{R}^{n}\right) \text { for } k\right. \\
& \left.=1,2, \ldots, n, \sqrt{V} u \in L^{2}\left(\mathbb{R}^{n}\right)\right\} .
\end{aligned}
$$

It is well known that this symmetric form is closed. Note also that it was shown by Simon [1] that this form coincides with the minimal closure of the form given by the same expression but defined on $C_{0}^{\infty}\left(\mathbb{R}^{n}\right)$ (the space of $C^{\infty}$ functions with compact supports). In other words, $C_{0}^{\infty}\left(\mathbb{R}^{n}\right)$ is a core of the form $Q$.
Let us denote by $L$ the self-adjoint operator associated with $Q$. The domain of $L$ is given by

$$
\begin{aligned}
& \mathscr{D}(L):=\left\{u \in \mathscr{V}: \exists v \in L^{2}\left(\mathbb{R}^{n}\right) \text { such that } \mathscr{Q}(u, \varphi)\right. \\
& \left.=\int_{\mathbb{R}^{n}} v \cdot \varphi d x, \forall \varphi \in \mathscr{V}\right\} .
\end{aligned}
$$

Formally, we write $L=-\Delta+V$ as a Schrödinger operator with potential $V$. Let $\left\{e^{-t L}\right\}_{t>0}$ be the semigroup of linear operators generated by $-L$ and let $p_{t}(x, y)$ be their kernels. Since $V$ is a locally integrable nonnegative function on $\mathbb{R}^{n}$, then the Feynman-Kac formula implies that the semigroup kernels $p_{t}(x, y)$ associated with $e^{-t L}$ satisfy the estimates:

$$
0 \leq p_{t}(x, y) \leq \frac{1}{(4 \pi t)^{n / 2}} \exp \left\{-\frac{|x-y|^{2}}{4 t}\right\}
$$

for all $t>0$ and all $x, y \in \mathbb{R}^{n}$.

Since the Schrödinger operator $L$ is a self-adjoint and positive definite operator acting on $L^{2}\left(\mathbb{R}^{n}\right)$, then $L$ admits the following spectral decomposition:

$$
L=\int_{0}^{\infty} \lambda d E_{L}(\lambda)
$$


where $E_{L}(\lambda)$ denotes its spectral resolution. For any $\gamma \in \mathbb{R}$, we can define the imaginary power $L^{i \gamma}$ associated with $L$ by the formula

$$
L^{i \gamma}:=\int_{0}^{\infty} \lambda^{i \gamma} d E_{L}(\lambda)
$$

By the functional calculus for $L$, we can also define the operator $L^{i \gamma}$ as follows:

$$
L^{i \gamma}(f)(x):=\frac{1}{\Gamma(-i \gamma)} \int_{0}^{\infty} t^{-i \gamma-1} e^{-t L}(f)(x) d t .
$$

By the spectral theory, we know that $\left\|L^{i \gamma}\right\|_{L^{2} \rightarrow L^{2}}=1$ for all $\gamma \in \mathbb{R}$. Moreover, it was proved by Shen [2] that $L^{i \gamma}$ is a Calderón-Zygmund operator provided that $V \in$ $\mathrm{RH}_{n / 2}$ (reverse Hölder class). We refer the reader to [2-4] for related results concerning the imaginary powers of selfadjoint operators.

For any $0<\alpha<n$, the fractional integrals $L^{-\alpha / 2}$ associated with $L$ are defined by

$$
L^{-\alpha / 2}(f)(x):=\frac{1}{\Gamma(\alpha / 2)} \int_{0}^{\infty} t^{\alpha / 2-1} e^{-t L}(f)(x) d t .
$$

Since the kernel $p_{t}(x, y)$ of $\left\{e^{-t L}\right\}_{t>0}$ satisfies the Gaussian upper bounds (4), then it is easy to check that $\left|L^{-\alpha / 2}(f)(x)\right| \leq$ $C \cdot I_{\alpha}(|f|)(x)$ for all $x \in \mathbb{R}^{n}$, where $I_{\alpha}$ denotes the classical fractional integral operator (see $[5]$ ):

$$
I_{\alpha}(f)(x):=\frac{\Gamma((n-\alpha) / 2)}{2^{\alpha} \pi^{n / 2} \Gamma(\alpha / 2)} \int_{\mathbb{R}^{n}} \frac{f(y)}{|x-y|^{n-\alpha}} d y .
$$

Hence, by using the $L^{p}-L^{q}$ boundedness of $I_{\alpha}$ (see [5]), we have

$$
\left\|L^{-\alpha / 2}(f)\right\|_{L^{q}} \leq C\left\|I_{\alpha}(f)\right\|_{L^{q}} \leq C\|f\|_{L^{p}},
$$

where $1<p<n / \alpha$ and $1 / q=1 / p-\alpha / n$. For more information about the fractional integrals $L^{-\alpha / 2}$ associated with some classes of operators, one can see [6-9].

In [10], Song and Yan introduced the weighted Hardy space $H_{L}^{1}(w)$ associated with $L$ in terms of the square function and established its atomic decomposition theory. Furthermore, they also showed that the Riesz transform $\nabla L^{-1 / 2}$ associated with $L$ is bounded on $L^{p}(w)$ for $1<p<2$ and bounded from $H_{L}^{1}(w)$ to the classical weighted Hardy space $H^{1}(w)$ (see $[11,12]$ for $\left.H^{1}(w)\right)$.

Recently, in [13], we defined the weighted Hardy spaces $H_{L}^{p}(w)$ associated with $L$ for $0<p<1$ and gave their atomic decompositions. We also obtained that $\nabla L^{-1 / 2}$ is bounded from $H_{L}^{p}(w)$ to the classical weighted Hardy space $H^{p}(w)$ (see also [11, 12] for $\left.H^{p}(w)\right)$ when $n /(n+1)<p<1$. In this paper, we first define molecules for the weighted Hardy spaces $H_{L}^{p}(w)$ associated with $L$ and then establish their molecular characterizations. As applications of the molecular characterization combining with the atomic decomposition of $H_{L}^{p}(w)$, we will obtain some estimates of $L^{i \gamma}$ and $L^{-\alpha / 2}$ on $H_{L}^{p}(w)$ for $n /(n+1)<p \leq 1$. Our main results are stated as follows.
Theorem 1. Let $L=-\Delta+V, n /(n+1)<p \leq 1$, and $w \epsilon$ $A_{1} \cap R H_{(2 / p)^{\prime}}$. Then, for any $\gamma \in \mathbb{R}$, the imaginary power $L^{i \gamma}$ is bounded from $H_{L}^{p}(w)$ to the weighted Lebesgue space $L^{p}(w)$.

Theorem 2. Let $L=-\Delta+V, n /(n+1)<p \leq 1$, and $w \in$ $A_{1} \cap R H_{(2 / p)^{\prime}}$. Then, for any $\gamma \in \mathbb{R}$, the imaginary power $L^{i \gamma}$ is bounded on $H_{L}^{p}(w)$.

Theorem 3. Suppose that $L=-\Delta+V$. Let $0<\alpha<n / 2$, $n /(n+1)<p \leq 1,1 / q=1 / p-\alpha / n$, and $w \in A_{1} \cap R H_{(2 / p)^{\prime}}$. Then, the fractional integral operator $L^{-\alpha / 2}$ is bounded from $H_{L}^{p}(w)$ to $L^{q}\left(w^{q / p}\right)$.

Theorem 4. Suppose that $L=-\Delta+V$. Let $0<\alpha<$ $\min \{n / 2,1\}, n /(n+1)<p \leq n /(n+\alpha), 1 / q=1 / p-\alpha / n$, and $w \in A_{1} \cap R H_{(2 / p)^{\prime}}$. Then, the fractional integral operator $L^{-\alpha / 2}$ is bounded from $H_{L}^{p}(w)$ to $H_{L}^{q}\left(w^{q / p}\right)$.

\section{Notations and Preliminaries}

Let us first recall some standard definitions and notations. The classical $A_{p}$ weight theory was first introduced by Muckenhoupt in the study of weighted $L^{p}$ boundedness of Hardy-Littlewood maximal functions in [14]. A weight $w$ is a nonnegative, locally integrable function defined on $\mathbb{R}^{n}$, and $B=B\left(x_{0}, r_{B}\right)$ denotes the ball with the center $x_{0}$ and radius $r_{B}$. We say that $w \in A_{1}$, if

$$
\frac{1}{|B|} \int_{B} w(x) d x \leq C \cdot \underset{x \in B}{\operatorname{essinf}} w(x),
$$

for every ball $B \subseteq \mathbb{R}^{n}$,

where $C$ is a positive constant which is independent of $B$. A weight function $w$ is said to belong to the reverse Hölder class $\mathrm{RH}_{s}$, if there exist two constants $s>1$ and $\mathrm{C}>0$ such that the following reverse Hölder inequality holds:

$$
\begin{aligned}
\left(\frac{1}{|B|} \int_{B} w(x)^{s} d x\right)^{1 / s} \leq C\left(\frac{1}{|B|} \int_{B} w(x) d x\right), & \\
& \text { for every ball } B \subseteq \mathbb{R}^{n} .
\end{aligned}
$$

Given a ball $B$ and $\lambda>0, \lambda B$ denotes the ball with the same center as $B$ whose radius is $\lambda$ times that of $B$. For a given weight function $w$ and a measurable set $E$, we denote the Lebesgue measure of $E$ by $|E|$ and the weighted measure of $E$ by $w(E)$, where $w(E)=\int_{E} w(x) d x$.

We give the following results which will be often used in the sequel.

Lemma 5 (see [15]). Let $w \in A_{1}$. Then, for any ball $B$, there exists an absolute constant $C>0$ such that

$$
w(2 B) \leq C w(B) .
$$

In general, for any $\lambda>1$, we have

$$
w(\lambda B) \leq C \cdot \lambda^{n} w(B),
$$

where $C$ does not depend on $B$ nor on $\lambda$. 
Lemma 6 (see $[15])$. Let $w \in A_{1}$. Then, there exists a constant $C>0$ such that

$$
C \cdot \frac{|E|}{|B|} \leq \frac{w(E)}{w(B)}
$$

for any measurable subset $E$ of a ball $B$.

Given a weight function $w$ on $\mathbb{R}^{n}$, for $0<p<\infty$, we denote by $L^{p}(w)$ the weighted space of all functions $f$ satisfying

$$
\|f\|_{L^{p}(w)}:=\left(\int_{\mathbb{R}^{n}}|f(x)|^{p} w(x) d x\right)^{1 / p}<\infty .
$$

In particular, when $w$ equals a constant function, we will denote $L^{p}(w)$ simply by $L^{p}\left(\mathbb{R}^{n}\right)$ and define

$$
\|f\|_{L^{p}}:=\left(\int_{\mathbb{R}^{n}}|f(x)|^{p} d x\right)^{1 / p}<\infty .
$$

Throughout this paper, we will use $C$ to denote a positive constant, which is independent of the main parameters and not necessarily the same at each occurrence. By $A \sim B$, we mean that there exists a constant $C>1$ such that $1 / C \leq$ $A / B \leq C$. Moreover, we denote the conjugate exponent of $s>1$ by $s^{\prime}=s /(s-1)$.

\section{Atomic Decomposition and Molecular Characterization of Weighted Hardy Spaces}

Let $L=-\Delta+V$. For any $t>0$, we define $P_{t}=e^{-t L}$ and

$$
Q_{t, k}:=\left.(-t)^{k} \frac{d^{k} P_{s}}{d s^{k}}\right|_{s=t}=(t L)^{k} e^{-t L}, \quad k=1,2, \ldots
$$

We denote $Q_{t, k}$ simply by $Q_{t}$ when $k=1$. First note that Gaussian upper bounds carry over from heat kernels to their time derivatives.

Lemma 7 (see $[16,17])$. For every $k=1,2, \ldots$, there exist two positive constants $C_{k}$ and $c_{k}$ such that the kernel $p_{t, k}(x, y)$ of the operator $Q_{t, k}$ satisfies

$$
\left|p_{t, k}(x, y)\right| \leq \frac{C_{k}}{(4 \pi t)^{n / 2}} \exp \left\{-\frac{|x-y|^{2}}{c_{k} t}\right\}
$$

for all $t>0$ and almost all $x, y \in \mathbb{R}^{n}$.

$$
\begin{aligned}
& \text { Let } \mathbb{R}_{+}^{n+1}=\mathbb{R}^{n} \times(0, \infty) \text {. For any } x \in \mathbb{R}^{n} \text {, we set } \\
& \qquad \Gamma(x)=\left\{(y, t) \in \mathbb{R}_{+}^{n+1}:|x-y|<t\right\} .
\end{aligned}
$$

For a given function $f \in L^{2}\left(\mathbb{R}^{n}\right)$, we consider the square function associated with Schrödinger operator $L$, which is defined by (see $[18,19])$

$$
S_{L}(f)(x):=\left(\iint_{\Gamma(x)}\left|Q_{t^{2}}(f)(y)\right|^{2} \frac{d y d t}{t^{n+1}}\right)^{1 / 2},
$$

Set

$$
H_{L}^{2}\left(\mathbb{R}^{n}\right)=\overline{\mathscr{R}(L)}=\overline{\left\{L u \in L^{2}\left(\mathbb{R}^{n}\right): u \in L^{2}\left(\mathbb{R}^{n}\right)\right\}},
$$

where $\mathscr{R}(L)$ stands for the range of $L$ in $L^{2}\left(\mathbb{R}^{n}\right)$. Given a weight function $w$ on $\mathbb{R}^{n}$, in $[10,13]$, the authors defined the weighted Hardy spaces $H_{L}^{p}(w)$ for $0<p \leq 1$ as the completion of $H_{L}^{2}\left(\mathbb{R}^{n}\right)$ in the norm given by the $L^{p}(w)$ quasinorm of square functions; that is,

$$
\|f\|_{H_{L}^{p}(w)}:=\left\|S_{L}(f)\right\|_{L^{p}(w)} .
$$

For the Schrödinger operator $L=-\Delta+V$, it can be shown that $H_{L}^{2}\left(\mathbb{R}^{n}\right)=L^{2}\left(\mathbb{R}^{n}\right)$ (see, e.g., [19]). In [10], Song and Yan characterized weighted Hardy spaces $H_{L}^{1}(w)$ in terms of atoms in the following way and obtained their atomic characterizations.

Definition 8 (see [10]). Let $M \in \mathbb{N}$ and $p=1$. A function $a(x) \in L^{2}\left(\mathbb{R}^{n}\right)$ is called a $(1,2, M)$-atom with respect to $w$ (or a $w$-(1,2,M)-atom) if there exist a ball $B=B\left(x_{0}, r_{B}\right)$ and a function $b \in \mathscr{D}\left(L^{M}\right)$ such that
(a) $a=L^{M} b$;
(b) $\operatorname{supp} L^{k} b \subseteq B, k=0,1, \ldots, M$;
(c) $\left\|\left(r_{B}^{2} L\right)^{k} b\right\|_{L^{2}(B)} \leq r_{B}^{2 M}|B|^{1 / 2}[w(B)]^{-1}, k=0,1, \ldots, M$.

Theorem 9 (see [10]). Let $M \in \mathbb{N}$ and $w \in A_{1} \cap R H_{2}$. If $f \in H_{L}^{1}(w) \cap L^{2}\left(\mathbb{R}^{n}\right)$, then there exist a family of $w-(1,2, M)$ atoms $\left\{a_{j}\right\}$ and a sequence of real numbers $\left\{\lambda_{j}\right\}$ with $\sum_{j}\left|\lambda_{j}\right| \leq$ $C\|f\|_{H_{L}^{1}(w)}$ such that $f$ can be represented in the form $f(x)=$ $\sum_{j} \lambda_{j} a_{j}(x)$, and the sum converges in the sense of both $L^{2}\left(\mathbb{R}^{n}\right)$ norm and $H_{L}^{1}(w)$-norm.

Similarly, in [13], we introduced the notion of weighted atoms for $H_{L}^{p}(w)(0<p<1)$ and proved their atomic characterizations.

Definition 10 (see [13]). Let $M \in \mathbb{N}$ and $0<p<1$. A function $a(x) \in L^{2}\left(\mathbb{R}^{n}\right)$ is called a $(p, M ; L)$-atom with respect to $w$ (or a $w$ - $(p, M ; L)$-atom) if there exist a ball $B=B\left(x_{0}, r_{B}\right)$ and a function $b \in \mathscr{D}\left(L^{M}\right)$ such that

$$
\begin{aligned}
& \left(\mathrm{a}^{\prime}\right) a=L^{M} b \\
& \left(\mathrm{~b}^{\prime}\right) \operatorname{supp} L^{k} b \subseteq B, k=0,1, \ldots, M \\
& \left(\mathrm{c}^{\prime}\right)\left\|\left(r_{B}^{2} L\right)^{k} b\right\|_{L^{2}(B)} \leq r_{B}^{2 M}|B|^{1 / 2}[w(B)]^{-1 / p}, k=0,1, \ldots, M .
\end{aligned}
$$

Theorem 11 (see [13]). Let $M \in \mathbb{N}$ and $0<p<1$.

(i) If $w \in A_{1}$ and $f \in H_{L}^{p}(w) \cap L^{2}\left(\mathbb{R}^{n}\right)$, then there exist a family of $w$ - $(p, M ; L)$-atoms $\left\{a_{j}\right\}$ and a sequence of real numbers $\left\{\lambda_{j}\right\}$ with $\sum_{j}\left|\lambda_{j}\right|^{p} \leq C\|f\|_{H_{L}^{p}(w)}^{p}$ such that $f$ can be represented in the form $f(x)=\sum_{j} \lambda_{j} a_{j}(x)$, and the sum converges in the sense of both $L^{2}\left(\mathbb{R}^{n}\right)$-norm and $H_{L}^{p}(w)$-norm. 
(ii) If $w \in A_{1} \cap R H_{(2 / p)^{\prime}}$ and $n /(n+1)<p<1$, then every $w$ - $(p, M ; L)$-atom $a$ is in $H_{L}^{p}(w)$ and its $H_{L}^{p}(w)$-norm is uniformly bounded; that is, there exists a constant $C>0$ independent of a such that $\|a\|_{H_{L}^{p}(w)} \leq C$.

For every bounded Borel function $F:[0, \infty) \rightarrow \mathbb{C}$, we define the operator $F(L): L^{2}\left(\mathbb{R}^{n}\right) \rightarrow L^{2}\left(\mathbb{R}^{n}\right)$ by the following formula:

$$
F(L):=\int_{0}^{\infty} F(\lambda) d E_{L}(\lambda)
$$

where $E_{L}(\lambda)$ is the spectral decomposition of $L$. Therefore, the operator $\cos (t \sqrt{L})$ is well defined on $L^{2}\left(\mathbb{R}^{n}\right)$. Moreover, it follows from [20] that there exists a constant $c_{0}$ such that the Schwartz kernel $K_{\cos (t \sqrt{L})}(x, y)$ of $\cos (t \sqrt{L})$ has support contained in $\left\{(x, y) \in \mathbb{R}^{n} \times \mathbb{R}^{n}:|x-y| \leq c_{0} t\right\}$. By the functional calculus for $L$ and Fourier inversion formula, whenever $F$ is an even bounded Borel function with $\widehat{F} \in$ $L^{1}(\mathbb{R})$, we can write $F(\sqrt{L})$ in terms of $\cos (t \sqrt{L})$. More precisely

$$
F(\sqrt{L})=(2 \pi)^{-1} \int_{-\infty}^{\infty} \widehat{F}(t) \cos (t \sqrt{L}) d t
$$

which gives

$$
\begin{aligned}
& K_{F(\sqrt{L})}(x, y) \\
& \quad=(2 \pi)^{-1} \int_{|t| \geq c_{0}^{-1}|x-y|} \widehat{F}(t) K_{\cos (t \sqrt{L})}(x, y) d t .
\end{aligned}
$$

Lemma 12 (see $[10,19])$. Let $\varphi \in C_{0}^{\infty}(\mathbb{R})$ be even and $\operatorname{supp} \varphi \subseteq$ $\left[-c_{0}^{-1}, c_{0}^{-1}\right]$. Let $\Phi$ denote the Fourier transform of $\varphi$. Then, for each $j=0,1, \ldots$ and for all $t>0$, the Schwartz kernel $K_{\left(t^{2} L\right)^{j} \Phi(t \sqrt{L})}(x, y)$ of $\left(t^{2} L\right)^{j} \Phi(t \sqrt{L})$ satisfies

$$
\operatorname{supp} K_{\left(t^{2} L\right)^{j} \Phi(t \sqrt{L})} \subseteq\left\{(x, y) \in \mathbb{R}^{n} \times \mathbb{R}^{n}:|x-y| \leq t\right\} .
$$

For a given real number $s>0$, we define

$$
\begin{aligned}
\mathscr{F}(s) & :=\{\psi: \mathbb{C} \longrightarrow \mathbb{C} \text { measurable, }|\psi(z)| \leq C \\
& \left.\frac{|z|^{s}}{1+|z|^{2 s}}\right\} .
\end{aligned}
$$

Then, for any nonzero function $\psi \in \mathscr{F}(s)$, we can prove the following estimate (see $[10,19])$ :

$$
\left(\int_{0}^{\infty}\|\psi(t \sqrt{L}) f\|_{L^{2}}^{2} \frac{d t}{t}\right)^{1 / 2}=C\|f\|_{L^{2}},
$$

where $C=\left(\int_{0}^{\infty}|\psi(t)|^{2}(d t / t)\right)^{1 / 2}$. Inspired by $[19,21,22]$, we are now going to define the weighted molecules corresponding to the weighted atoms mentioned above.
Definition 13. Let $\varepsilon>0, M \in \mathbb{N}$, and $0<p \leq 1$. A function $m(x) \in L^{2}\left(\mathbb{R}^{n}\right)$ is called a $w$ - $(p, M, \varepsilon ; L)$-molecule associated with $L$, if there exist a ball $B=B\left(x_{0}, r_{B}\right)$ and a function $b \in$ $\mathscr{D}\left(L^{M}\right)$ such that
(A) $m=L^{M} b$;
(B) $\left\|\left(r_{B}^{2} L\right)^{k} b\right\|_{L^{2}(2 B)} \leq r_{B}^{2 M}|B|^{1 / 2}[w(B)]^{-1 / p}, k=0,1, \ldots$, $M$;
(C) $\left\|\left(r_{B}^{2} L\right)^{k} b\right\|_{L^{2}\left(2^{j+1} B \backslash 2^{j} B\right)} \leq 2^{-j \varepsilon} r_{B}^{2 M}\left|2^{j} B\right|^{1 / 2}\left[w\left(2^{j} B\right)\right]^{-1 / p}$, $k=0,1, \ldots, M, j=1,2, \ldots$.

Clearly, for every $w$ - $(p, M ; L)$-atom $a$, it is also a $w$ $(p, M, \varepsilon ; L)$-molecule for all $\varepsilon>0$. Then, we are able to establish the following molecular characterizations for the weighted Hardy spaces $H_{L}^{p}(w)(0<p \leq 1)$ associated with L.

Theorem 14. Let $\varepsilon>0, M \in \mathbb{N}$, and $0<p \leq 1$.

(i) If $f \in H_{L}^{p}(w) \cap L^{2}\left(\mathbb{R}^{n}\right)$ and $w \in A_{1}$, then there exist a family of $w$ - $(p, M, \varepsilon ; L)$-molecules $\left\{m_{j}\right\}$ and a sequence of real numbers $\left\{\lambda_{j}\right\}$ with $\sum_{j}\left|\lambda_{j}\right|^{p} \leq C\|f\|_{H_{L}^{p}(w)}^{p}$ such that $f(x)=\sum_{j} \lambda_{j} m_{j}(x)$, and the sum converges in the sense of both $L^{2}\left(\mathbb{R}^{n}\right)$-norm and $H_{L}^{p}(w)$-norm.

(ii) Assume that $M>(n / 2)(1 / p-1 / 2)$ and $w \in A_{1} \cap$ $R H_{(2 / p)^{\prime}}$. Then, every $w$ - $(p, M, \varepsilon ; L)$-molecule $m$ is in $H_{L}^{p}(w)$. Moreover, there exists a constant $C>0$ independent of $m$ such that $\|m\|_{H_{L}^{p}(w)} \leq C$.

Proof. (i) This is a straightforward consequence of Theorems 9 and 11. (ii) We will use some ideas from [19, 22]. Suppose that $m$ is a $w$ - $(p, M, \varepsilon ; L)$-molecule associated with a ball $B=B\left(x_{0}, r_{B}\right)$. Let $\varphi \in C_{0}^{\infty}(\mathbb{R})$ be even with $\operatorname{supp} \varphi \subseteq$ $\left[-\left(2 c_{0}\right)^{-1},\left(2 c_{0}\right)^{-1}\right]$ and let $\Phi$ denote the Fourier transform of $\varphi$. We set $\Psi(x)=x^{2} \Phi(x), x \in \mathbb{R}$. By the $L^{2}$-functional calculus of $L$, for every $m \in L^{2}\left(\mathbb{R}^{n}\right)$, we can establish the following version of the Calderón reproducing formula:

$$
m(x)=\mathcal{c}_{\Psi} \int_{0}^{\infty}\left(t^{2} L\right)^{M} \Psi^{2}(t \sqrt{L})(m)(x) \frac{d t}{t},
$$

where the above equality holds in the sense of $L^{2}\left(\mathbb{R}^{n}\right)$-norm. Set

$$
\begin{aligned}
& U_{0}(B)=2 B, \\
& U_{j}(B)=2^{j+1} B \backslash 2^{j} B, \quad j=1,2, \ldots .
\end{aligned}
$$

Then, we can decompose

$$
\begin{aligned}
\mathbb{R}^{n} \times(0, \infty)= & \left(\bigcup_{j=0}^{\infty} U_{j}(B) \times\left(0,2^{j} r_{B}\right]\right) \\
& \cup\left(\bigcup_{j=1}^{\infty} 2^{j} B \times\left(2^{j-1} r_{B}, 2^{j} r_{B}\right]\right) .
\end{aligned}
$$


For any measurable set $E$ in $\mathbb{R}^{n}$, we denote $\chi_{E}$ the characteristic function of the set $E$. Hence, by formula (30), we are able to write

$$
\begin{aligned}
& m(x) \\
& =c_{\Psi} \sum_{j=0}^{\infty} \int_{0}^{2^{j} r_{B}}\left(t^{2} L\right)^{M} \Psi^{2}(t \sqrt{L})\left(m \chi_{U_{j}(B)}\right)(x) \frac{d t}{t} \\
& \quad+c_{\Psi} \sum_{j=1}^{\infty} \int_{2^{j-1} r_{B}}^{2^{j} r_{B}}\left(t^{2} L\right)^{M} \Psi^{2}(t \sqrt{L})\left(m \chi_{2^{j} B}\right)(x) \frac{d t}{t} \\
& :=\sum_{j=0}^{\infty} m_{j}^{(1)}(x)+\sum_{j=1}^{\infty} m_{j}^{(2)}(x) .
\end{aligned}
$$

Let us first consider the terms $\left\{m_{j}^{(1)}\right\}_{j=0}^{\infty}$. We will show that each $m_{j}^{(1)}$ is a multiple of a $w$ - $(p, M ; L)$-atom with a sequence of coefficients in $\ell^{p}$. Indeed, for every $j=0,1,2, \ldots$, one can write

$$
m_{j}^{(1)}(x)=L^{M} b_{j}(x),
$$

where

$$
b_{j}(x)=\mathcal{c}_{\Psi} \int_{0}^{2^{j} r_{B}} t^{2 M} \Psi^{2}(t \sqrt{L})\left(m \chi_{U_{j}(B)}\right)(x) \frac{d t}{t} .
$$

By using Lemma 12, we can easily conclude that, for every $k=$ $0,1, \ldots, M, \operatorname{supp}\left(L^{k} b_{j}\right) \subseteq 2^{j+1} B$. In addition, by the duality argument, we get

$$
\begin{aligned}
& \left\|\left[\left(2^{j+1} r_{B}\right)^{2} L\right]^{k} b_{j}\right\|_{L^{2}\left(2^{j+1} B\right)} \\
& =\sup _{\|h\|_{L^{2}\left(2^{j+1} B\right)} \leq 1}\left|\int_{2^{j+1} B}\left[\left(2^{j+1} r_{B}\right)^{2} L\right]^{k} b_{j}(x) h(x) d x\right| .
\end{aligned}
$$

Then, it follows from Hölder's inequality and estimate (29) that

$$
\begin{aligned}
& \left|\int_{2^{j+1} B}\left[\left(2^{j+1} r_{B}\right)^{2} L\right]^{k} b_{j}(x) h(x) d x\right|=c_{\Psi}\left(2^{j+1} r_{B}\right)^{2 k} \\
& \cdot \mid \int_{0}^{2^{j} r_{B}} \int_{2^{j+1} B} t^{2 M} L^{k} \Psi(t \sqrt{L})\left(m \chi_{U_{j}(B)}\right)(y) \\
& \cdot \Psi(t \sqrt{L})(h)(y) \frac{d y d t}{t} \mid \leq c_{\Psi}\left(2^{j+1} r_{B}\right)^{2 k} \\
& \cdot\left(2^{j} r_{B}\right)^{2 M-2 k} \mid \int_{0}^{2^{j} r_{B}} \int_{2^{j+1} B}\left(t^{2} L\right)^{k} \Psi(t \sqrt{L}) \\
& \cdot\left(m \chi_{U_{j}(B)}\right)(y) \cdot \Psi(t \sqrt{L})(h)(y) \frac{d y d t}{t} \mid
\end{aligned}
$$

$$
\begin{aligned}
& \leq c_{\Psi}\left(2^{j+1} r_{B}\right)^{2 M}\left(\int_{0}^{\infty} \|\left(t^{2} L\right)^{k} \Psi(t \sqrt{L})\right. \\
& \left.\cdot\left(m \chi_{U_{j}(B)}\right) \|_{L^{2}}^{2} \frac{d t}{t}\right)^{1 / 2}\left(\int_{0}^{\infty} \| \Psi(t \sqrt{L})\right. \\
& \left.\cdot\left(h \chi_{2^{j+1} B}\right) \|_{L^{2}}^{2} \frac{d t}{t}\right)^{1 / 2} \leq c_{\Psi}\left(2^{j+1} r_{B}\right)^{2 M} \\
& \cdot\left\|m \chi_{U_{j}(B)}\right\|_{L^{2}} \cdot\left\|h \chi_{2^{j+1} B}\right\|_{L^{2}} \leq C \cdot 2^{-j \varepsilon}\left(2^{j+1} r_{B}\right)^{2 M} \\
& \cdot\left|2^{j+1} B\right|^{1 / 2}\left[w\left(2^{j+1} B\right)\right]^{-1 / p} .
\end{aligned}
$$

Hence,

$$
\begin{aligned}
& \left\|\left[\left(2^{j+1} r_{B}\right)^{2} L\right]^{k} b_{j}\right\|_{L^{2}\left(2^{j+1} B\right)} \\
& \quad \leq C \cdot 2^{-j \varepsilon}\left(2^{j+1} r_{B}\right)^{2 M}\left|2^{j+1} B\right|^{1 / 2}\left[w\left(2^{j+1} B\right)\right]^{-1 / p},
\end{aligned}
$$

which implies our desired result. Next we consider the terms $\left\{m_{j}^{(2)}\right\}_{j=1}^{\infty}$. For every $j=1,2, \ldots$, we write

$$
\begin{aligned}
m_{j}^{(2)}(x) & \\
= & c_{\Psi} \int_{2^{j-1} r_{B}}^{2^{j} r_{B}}\left(t^{2} L\right)^{M} \Psi^{2}(t \sqrt{L})(m)(x) \frac{d t}{t} \\
& \quad-c_{\Psi} \int_{2^{j-1} r_{B}}^{2^{j} r_{B}}\left(t^{2} L\right)^{M} \Psi^{2}(t \sqrt{L})\left(m \chi_{\left(2^{j} B\right)^{c}}\right)(x) \frac{d t}{t} \\
& :=m_{j}^{(21)}(x)-m_{j}^{(22)}(x) .
\end{aligned}
$$

To deal with the term $m_{j}^{(21)}$, we recall that $m=L^{M} b$ for some $b \in \mathscr{D}\left(L^{M}\right)$, and then

$$
\begin{aligned}
m_{j}^{(21)}(x) & =c_{\Psi} \int_{2^{j-1} r_{B}}^{2^{j} r_{B}}\left(t^{2} L\right)^{M} \Psi^{2}(t \sqrt{L})\left(L^{M} b\right)(x) \frac{d t}{t} \\
& =L^{M} b_{j}^{(21)}(x),
\end{aligned}
$$

where

$$
b_{j}^{(21)}(x)=c_{\Psi} \int_{2^{j-1} r_{B}}^{2^{j} r_{B}}\left(t^{2} L\right)^{M} \Psi^{2}(t \sqrt{L})(b)(x) \frac{d t}{t} .
$$

Since $b(x)=b(x) \chi_{2^{j} B}(x)+\sum_{l=j}^{\infty} b(x) \chi_{U_{l}(B)}(x)$, then we can further write

$$
b_{j}^{(21)}(x)=b_{1, j}^{(21)}(x)+\sum_{l=j}^{\infty} b_{l j}^{(21)}(x),
$$


6

Journal of Function Spaces

where

$$
\begin{aligned}
& b_{1, j}^{(21)}(x)=\mathcal{c}_{\Psi} \int_{2^{j-1} r_{B}}^{2^{j} r_{B}}\left(t^{2} L\right)^{M} \Psi^{2}(t \sqrt{L})\left(b \chi_{2^{j} B}\right)(x) \frac{d t}{t}, \\
& b_{l j}^{(21)}(x) \\
& =c_{\Psi} \int_{2^{j-1} r_{B}}^{2^{j} r_{B}}\left(t^{2} L\right)^{M} \Psi^{2}(t \sqrt{L})\left(b \chi_{U_{l}(B)}\right)(x) \frac{d t}{t} .
\end{aligned}
$$

By using Lemma 12 again, we have $\operatorname{supp}\left(L^{k} b_{1, j}^{(21)}\right) \subseteq 2^{j} B$ and $\operatorname{supp}\left(L^{k} b_{l j}^{(21)}\right) \subseteq 2^{l+1} B$ for every $k=0,1, \ldots, M$. Moreover, it follows from Minkowski's integral inequality that

$$
\begin{aligned}
& \left\|\left[\left(2^{j} r_{B}\right)^{2} L\right]^{k} b_{1, j}^{(21)}\right\|_{L^{2}\left(2^{j} B\right)}=\mathcal{c}_{\Psi}\left(2^{j} r_{B}\right)^{2 k} \\
& \cdot\left\|\int_{2^{j-1} r_{B}}^{2^{j} r_{B}} t^{2 M} L^{M+k} \Psi^{2}(t \sqrt{L})\left(b \chi_{2^{j} B}\right) \frac{d t}{t}\right\|_{L^{2}\left(2^{j} B\right)} \\
& \leq \mathcal{C}_{\Psi}\left(2^{j} r_{B}\right)^{2 k} \\
& \cdot \int_{2^{j-1} r_{B}}^{2^{j} r_{B}}\left\|\left(t^{2} L\right)^{M+k} \Psi^{2}(t \sqrt{L})\left(b \chi_{2^{j} B}\right)\right\|_{L^{2}\left(2^{j} B\right)} \frac{d t}{t^{2 k+1}} \\
& \leq C\left\|b \chi_{2^{j} B}\right\|_{L^{2}\left(2^{j} B\right)} \leq C \sum_{l=0}^{j-1}\left\|b \chi_{U_{l}(B)}\right\|_{L^{2}\left(2^{j} B\right)} \\
& \quad \leq C \sum_{l=0}^{j-1} 2^{-l \varepsilon} r_{B}^{2 M}\left|2^{l} B\right|^{1 / 2}\left[w\left(2^{l} B\right)\right]^{-1 / p} .
\end{aligned}
$$

When $0 \leq l \leq j-1$, then $2^{l} B \subseteq 2^{j} B$. Since $w \in A_{1}$, then, by using Lemma 6, we can get

$$
\frac{w\left(2^{l} B\right)}{w\left(2^{j} B\right)} \geq C \cdot \frac{\left|2^{l} B\right|}{\left|2^{j} B\right|}
$$

Consequently,

$$
\begin{aligned}
& \left\|\left[\left(2^{j} r_{B}\right)^{2} L\right]^{k} b_{1, j}^{(21)}\right\|_{L^{2}\left(2^{j} B\right)} \leq C \cdot 2^{-j[2 M-n(1 / p-1 / 2)]} \\
& \cdot\left(2^{j} r_{B}\right)^{2 M}\left|2^{j} B\right|^{1 / 2}\left[w\left(2^{j} B\right)\right]^{-1 / p} \sum_{l=0}^{\infty} \frac{1}{2^{l \varepsilon}} \cdot \frac{1}{2^{l(n / p-n / 2)}} \\
& \leq C \cdot 2^{-j[2 M-n(1 / p-1 / 2)]} \cdot\left(2^{j} r_{B}\right)^{2 M}\left|2^{j} B\right|^{1 / 2} \\
& \cdot\left[w\left(2^{j} B\right)\right]^{-1 / p} \cdot
\end{aligned}
$$

On the other hand,

$$
\begin{aligned}
& \left\|\left[\left(2^{l+1} r_{B}\right)^{2} L\right]^{k} b_{l j}^{(21)}\right\|_{L^{2}\left(2^{l+1} B\right)}=c_{\Psi}\left(2^{l+1} r_{B}\right)^{2 k} \\
& \cdot\left\|\int_{2^{j-1} r_{B}}^{2^{j} r_{B}} t^{2 M} L^{M+k} \Psi^{2}(t \sqrt{L})\left(b \chi_{U_{l}(B)}\right) \frac{d t}{t}\right\|_{L^{2}\left(2^{l+1} B\right)} \\
& \leq c_{\Psi}\left(2^{l+1} r_{B}\right)^{2 k} \int_{2^{j-1} r_{B}}^{2^{j} r_{B}} \|\left(t^{2} L\right)^{M+k} \Psi^{2}(t \sqrt{L}) \\
& \cdot\left(b \chi_{U_{l}(B)}\right) \|_{L^{2}\left(2^{l+1} B\right)} \frac{d t}{t^{2 k+1}} \leq C\left(2^{l+1} r_{B}\right)^{2 k} \\
& \cdot\left\|b \chi_{U_{l}(B)}\right\|_{L^{2}\left(2^{l+1} B\right)} \cdot \frac{1}{\left(2^{j} r_{B}\right)^{2 k} \leq C \cdot 2^{-l \varepsilon}\left(2^{l+1} r_{B}\right)^{2 M}} \\
& \cdot\left|2^{l+1} B\right|^{1 / 2}\left[w\left(2^{l+1} B\right)\right]^{-1 / p} .
\end{aligned}
$$

Observe that $2 M>n(1 / p-1 / 2)$. Thus, from the above discussions, we have already proved that each $m_{j}^{(21)}$ is a multiple of a $w$ - $(p, M ; L)$-atom with a sequence of coefficients in $\ell^{p}$. Finally, we estimate the terms $\left\{m_{j}^{(22)}\right\}_{j=1}^{\infty}$. For every $j=1,2, \ldots$, we decompose $m_{j}^{(22)}$ as follows:

$$
\begin{aligned}
& m_{j}^{(22)}(x) \\
& =c_{\Psi} \sum_{l=j}^{\infty} \int_{2^{j-1} r_{B}}^{2^{j} r_{B}}\left(t^{2} L\right)^{M} \Psi^{2}(t \sqrt{L})\left(m \chi_{U_{l}(B)}\right)(x) \frac{d t}{t} \\
& =\sum_{l=j}^{\infty} L^{M} b_{l j}^{(22)}(x),
\end{aligned}
$$

where

$$
b_{l j}^{(22)}(x)=\mathcal{c}_{\Psi} \int_{2^{j-1} r_{B}}^{2^{j} r_{B}} t^{2 M} \Psi^{2}(t \sqrt{L})\left(m \chi_{U_{l}(B)}\right)(x) \frac{d t}{t} .
$$

It follows immediately from Lemma 12 that $\operatorname{supp}\left(L^{k} b_{l j}^{(22)}\right) \subseteq$ $2^{l+1} B$ for every $k=1,2, \ldots, M$ and $l \geq j$. Moreover,

$$
\begin{aligned}
& \left\|\left[\left(2^{l+1} r_{B}\right)^{2} L\right]^{k} b_{l j}^{(22)}\right\|_{L^{2}\left(2^{l+1} B\right)}=c_{\Psi}\left(2^{l+1} r_{B}\right)^{2 k} \\
& \cdot\left\|\int_{2^{j-1} r_{B}}^{2^{j} r_{B}} t^{2 M} L^{k} \Psi^{2}(t \sqrt{L})\left(m \chi_{U_{l}(B)}\right) \frac{d t}{t}\right\|_{L^{2}\left(2^{l+1} B\right)} \\
& \leq \mathcal{C}_{\Psi}\left(2^{l+1} r_{B}\right)^{2 k}\left(2^{l} r_{B}\right)^{2 M-2 k} \\
& \cdot \int_{2^{j-1} r_{B}}^{2^{j} r_{B}}\left\|\left(t^{2} L\right)^{k} \Psi^{2}(t \sqrt{L})\left(m \chi_{U_{l}(B)}\right)\right\|_{L^{2}\left(2^{l+1} B\right)} \frac{d t}{t} \\
& \quad \leq c_{\Psi}\left(2^{l+1} r_{B}\right)^{2 M}\left\|m \chi_{U_{l}(B)}\right\|_{L^{2}\left(2^{l+1} B\right)} \leq C \\
& \quad \cdot 2^{-l \varepsilon}\left(2^{l+1} r_{B}\right)^{2 M}\left|2^{l+1} B\right|^{1 / 2}\left[w\left(2^{l+1} B\right)\right]^{-1 / p} .
\end{aligned}
$$


Therefore, we have showed that each $m_{j}^{(22)}$ is also a multiple of a $w$ - $(p, M ; L)$-atom with a sequence of coefficients in $\ell^{p}$. This completes the proof of Theorem 14.

\section{Proof of Theorems 1 and 2}

Proof of Theorem 1. By the known result, we have that, for any $\gamma \in \mathbb{R}$, the operator $L^{i \gamma}$ is linear and bounded on $L^{2}\left(\mathbb{R}^{n}\right)$ (see, e.g., $[2,23])$. Since $H_{L}^{p}(\mathrm{w}) \cap L^{2}\left(\mathbb{R}^{n}\right)$ is dense in $H_{L}^{p}(w)$, then, by Theorems 9 and 11 and a standard density argument, it is enough for us to show that, for any $w$ - $(p, M ; L)$-atom $a$, $M \in \mathbb{N}$, there exists a constant $C>0$ independent of $a$ such that $\left\|L^{i \gamma}(a)\right\|_{L^{p}(w)} \leq C$. Let $a$ be a $w$ - $(p, M ; L)$-atom associated with a ball $B=B\left(x_{0}, r_{B}\right),\|a\|_{L^{2}(B)} \leq|B|^{1 / 2}[w(B)]^{-1 / p}$. We write

$$
\begin{aligned}
\left\|L^{i \gamma}(a)\right\|_{L^{p}(w)}^{p}= & \int_{2 B}\left|L^{i \gamma}(a)(x)\right|^{p} w(x) d x \\
& +\sum_{k=1}^{\infty} \int_{2^{k+1} B \backslash 2^{k} B}\left|L^{i \gamma}(a)(x)\right|^{p} w(x) d x \\
:= & I_{1}+I_{2} .
\end{aligned}
$$

We set $s=2 / p>1$. Note that $w \in \mathrm{RH}_{s^{\prime}}$, and then it follows from Hölder's inequality, the $L^{2}$ boundedness of $L^{i \gamma}$, and Lemma 5 that

$$
\begin{aligned}
I_{1} & \leq\left(\int_{2 B}\left|L^{i \gamma}(a)(x)\right|^{2} d x\right)^{p / 2}\left(\int_{2 B} w(x)^{s^{\prime}} d x\right)^{1 / s^{\prime}} \\
& \leq C\|a\|_{L^{2}(B)}^{p} \cdot \frac{w(2 B)}{|2 B|^{1 / s}} \leq C .
\end{aligned}
$$

On the other hand, for any $x \in 2^{k+1} B \backslash 2^{k} B, k=1,2, \ldots$, by expression (7), we can write

$$
\begin{aligned}
\left|L^{i \gamma}(a)(x)\right| \leq & C \int_{0}^{\infty}\left|e^{-t L}(a)(x)\right| \frac{d t}{t} \\
\leq & C \int_{0}^{r_{B}^{2}}\left|e^{-t L}(a)(x)\right| \frac{d t}{t} \\
& +C \int_{r_{B}^{2}}^{\infty}\left|e^{-t L}(a)(x)\right| \frac{d t}{t}:=\mathrm{I}+\mathrm{II} .
\end{aligned}
$$

For the term I, we observe that when $x \in 2^{k+1} B \backslash 2^{k} B, y \in B$, then $|x-y| \geq 2^{k-1} r_{B}$. Hence, by using Hölder's inequality and estimate (4), we deduce

$$
\begin{aligned}
\left|e^{-t L}(a)(x)\right| & \leq C \cdot \frac{\sqrt{t}}{\left(2^{k-1} r_{B}\right)^{n+1}} \int_{B}|a(y)| d y \\
& \leq C \cdot \frac{\sqrt{t}}{\left(2^{k} r_{B}\right)^{n+1}} \cdot\|a\|_{L^{2}(B)}|B|^{1 / 2} \\
& \leq C \cdot[w(B)]^{-1 / p} \cdot \frac{\sqrt{t}}{2^{k(n+1)} \cdot r_{B}} .
\end{aligned}
$$

So we have

$$
\begin{aligned}
\mathrm{I} & \leq C \cdot \frac{1}{2^{k(n+1)}[w(B)]^{1 / p}} \cdot \frac{1}{r_{B}} \int_{0}^{r_{B}^{2}} \frac{d t}{\sqrt{t}} \\
& \leq C \cdot \frac{1}{2^{k(n+1)}[w(B)]^{1 / p}} .
\end{aligned}
$$

We now turn to estimate the other term II. In this case, since there exists a function $b \in \mathscr{D}\left(L^{M}\right)$ such that $a=L^{M} b$ and $\|b\|_{L^{2}(B)} \leq r_{B}^{2 M}|B|^{1 / 2}[w(B)]^{-1 / p}$, then it follows from Hölder's inequality and Lemma 7 that

$$
\begin{aligned}
\left|e^{-t L}(a)(x)\right| & =\left|(t L)^{M} e^{-t L}(b)(x)\right| \cdot \frac{1}{t^{M}} \\
& \leq C \cdot \frac{1}{\left(2^{k-1} r_{B}\right)^{n+1}} \int_{B}|b(y)| d y \cdot \frac{1}{t^{M-1 / 2}} \\
& \leq C \cdot \frac{1}{\left(2^{k} r_{B}\right)^{n+1}}\|b\|_{L^{2}(B)}|B|^{1 / 2} \cdot \frac{1}{t^{M-1 / 2}} \\
& \leq C \cdot \frac{r_{B}^{2 M-1}}{2^{k(n+1)}[w(B)]^{1 / p}} \cdot \frac{1}{t^{M-1 / 2}} .
\end{aligned}
$$

Consequently,

$$
\begin{aligned}
\mathrm{II} & \leq C \cdot \frac{1}{2^{k(n+1)}[w(B)]^{1 / p}} \cdot r_{B}^{2 M-1} \int_{r_{B}^{2}}^{\infty} \frac{d t}{t^{M+1 / 2}} \\
& \leq C \cdot \frac{1}{2^{k(n+1)}[w(B)]^{1 / p}},
\end{aligned}
$$

where in the last inequality we have used the fact that $M \geq 1$. Therefore, by combining the above estimates for I and II, we obtain the following pointwise inequality:

$$
\begin{aligned}
&\left|L^{i \gamma}(a)(x)\right| \leq C \cdot \frac{1}{2^{k(n+1)}[w(B)]^{1 / p}}, \\
& \text { when } x \in 2^{k+1} B \backslash 2^{k} B .
\end{aligned}
$$

Notice that $w \in A_{1}$. Substituting inequality (58) into the term $I_{2}$ and using Lemma 5, then we have

$$
\begin{aligned}
I_{2} & \leq C \sum_{k=1}^{\infty} \frac{1}{2^{k p(n+1)} w(B)} \cdot w\left(2^{k+1} B\right) \\
& \leq C \sum_{k=1}^{\infty} \frac{1}{2^{k[p(n+1)-n]}} \leq C
\end{aligned}
$$

where the last series is convergent since $p>n /(n+1)$. Summarizing estimates (52) and (59) derived above, we complete the proof of Theorem 1 .

Proof of Theorem 2. Since $H_{L}^{p}(w) \cap L^{2}\left(\mathbb{R}^{n}\right)$ is dense in $H_{L}^{p}(w)$ and the operator $L^{i \gamma}$ is linear and bounded on $L^{2}\left(\mathbb{R}^{n}\right)$, then, in view of Theorems 9, 11, and 14, it suffices to verify that, for every $w$ - $(p, 2 M ; L)$-atom $a$, the function $m=L^{i \gamma}(a)$ is a multiple of a $w$ - $(p, M, \varepsilon ; L)$-molecule for some $\varepsilon>0$, and 
the multiplicative constant is independent of $a$. Let $a$ be a $w$-(p,2M;L)-atom with supp $a \subseteq B=B\left(x_{0}, r_{B}\right)$. By the definition, there exists a function $b \in \mathscr{D}\left(L^{2 M}\right)$ such that

$$
\begin{aligned}
a & =L^{2 M}(b) \\
\left\|\left(r_{B}^{2} L\right)^{k} b\right\|_{L^{2}(B)} \leq r_{B}^{4 M}|B|^{1 / 2}[w(B)]^{-1 / p}, & \\
k & =0,1, \ldots, 2 M .
\end{aligned}
$$

We set $\widetilde{b}=L^{i \gamma}\left(L^{M} b\right)$, and then $m=L^{M}(\widetilde{b})$. Obviously, we have $m(x) \in L^{2}\left(\mathbb{R}^{n}\right)$. Moreover, for $k=0,1, \ldots, M$, we can deduce

$$
\begin{aligned}
\left\|\left(r_{B}^{2} L\right)^{k} \tilde{b}\right\|_{L^{2}(2 B)} & =\frac{1}{r_{B}^{2 M}}\left\|L^{i \gamma}\left[\left(r_{B}^{2} L\right)^{M+k} b\right]\right\|_{L^{2}(2 B)} \\
& \leq C \cdot \frac{1}{r_{B}^{2 M}}\left\|\left(r_{B}^{2} L\right)^{M+k} b\right\|_{L^{2}(B)} \\
& \leq C \cdot r_{B}^{2 M}|B|^{1 / 2}[w(B)]^{-1 / p} .
\end{aligned}
$$

It remains to estimate $\left\|\left(r_{B}^{2} L\right)^{k} \widetilde{b}\right\|_{L^{2}\left(2^{j+1} B \mid 2^{j} B\right)}$ for $k=0,1, \ldots$, $M, j=1,2, \ldots$. We write

$$
\begin{aligned}
\left|\left(r_{B}^{2} L\right)^{k} \widetilde{b}(x)\right|= & \left|L^{i \gamma}\left[\left(r_{B}^{2} L\right)^{k} L^{M} b\right](x)\right| \\
\leq & C \int_{0}^{r_{B}^{2}}\left|e^{-t L}\left[r_{B}^{2 k} L^{M+k} b\right](x)\right| \frac{d t}{t} \\
& +C \int_{r_{B}^{2}}^{\infty}\left|e^{-t L}\left[r_{B}^{2 k} L^{M+k} b\right](x)\right| \frac{d t}{t} \\
:= & \mathrm{I}^{\prime}+\mathrm{II}^{\prime} .
\end{aligned}
$$

As mentioned in the proof of Theorem 1, we know that when $x \in 2^{j+1} B \backslash 2^{j} B, y \in B$, then $|x-y| \geq 2^{j-1} r_{B}, j=1,2, \ldots$ It follows from Hölder's inequality and estimate (4) that

$$
\begin{aligned}
I^{\prime} & \leq C \int_{0}^{r_{B}^{2}} \frac{\sqrt{t}}{\left(2^{j-1} r_{B}\right)^{n+1}}\left\|r_{B}^{2 k} L^{M+k} b\right\|_{L^{2}}|B|^{1 / 2} \frac{d t}{t} \leq C \\
& \cdot \frac{1}{\left(2^{j} r_{B}\right)^{n+1}}\left(\frac{1}{r_{B}^{2 M}} \cdot r_{B}^{4 M}|B|^{1 / 2}[w(B)]^{-1 / p}\right)|B|^{1 / 2} \\
& \cdot \int_{0}^{r_{B}^{2}} \frac{d t}{\sqrt{t}} \leq C \cdot \frac{1}{2^{j(n+1)}} \cdot r_{B}^{2 M}[w(B)]^{-1 / p} .
\end{aligned}
$$

Since $w \in A_{1}$ and $B \subseteq 2^{j} B, j=1,2, \ldots$, then, by using Lemma 6, we can get

$$
\frac{w(B)}{w\left(2^{j} B\right)} \geq C \cdot \frac{|B|}{\left|2^{j} B\right|} .
$$

Hence,

$$
\mathrm{I}^{\prime} \leq C \cdot \frac{1}{2^{j[(n+1)-n / p]}} \cdot r_{B}^{2 M}\left[w\left(2^{j} B\right)\right]^{-1 / p},
$$

Applying Hölder's inequality and Lemma 7, we obtain

$$
\begin{aligned}
\mathrm{II}^{\prime} \leq C \cdot r_{B}^{2 k} \int_{r_{B}^{2}}^{\infty}\left|(t L)^{M+k} e^{-t L}(b)(x)\right| \frac{d t}{t^{M+k+1}} \\
\leq C \cdot r_{B}^{2 k} \int_{r_{B}^{2}}^{\infty} \frac{1}{\left(2^{j-1} r_{B}\right)^{n+1}}\|b\|_{L^{2}(B)}|B|^{1 / 2} \frac{d t}{t^{M+k+1 / 2}} \\
\leq C \\
\quad \cdot \frac{1}{\left(2^{j} r_{B}\right)^{n+1}}\left(r_{B}^{4 M+2 k}|B|[w(B)]^{-1 / p}\right) \int_{r_{B}^{2}}^{\infty} \frac{d t}{t^{M+k+1 / 2}} \\
\leq C \cdot \frac{1}{2^{j(n+1)}} \cdot r_{B}^{2 M}[w(B)]^{-1 / p} .
\end{aligned}
$$

It follows immediately from inequality (64) that

$$
\mathrm{II}^{\prime} \leq C \cdot \frac{1}{2^{j[(n+1)-n / p]}} \cdot r_{B}^{2 M}\left[w\left(2^{j} B\right)\right]^{-1 / p},
$$

when $x \in 2^{j+1} B \backslash 2^{j} B$.

Combining the above estimates for $\mathrm{I}^{\prime}$ and $\mathrm{II}^{\prime}$, we thus obtain

$$
\begin{aligned}
& \left\|\left(r_{B}^{2} L\right)^{k} \widetilde{b}\right\|_{L^{2}\left(2^{j+1} B \backslash 2^{j} B\right)} \\
& \quad \leq C \cdot \frac{1}{2^{j[(n+1)-n / p]}} \cdot r_{B}^{2 M}\left|2^{j} B\right|^{1 / 2}\left[w\left(2^{j} B\right)\right]^{-1 / p} .
\end{aligned}
$$

Observe that $p>n /(n+1)$. If we set $\varepsilon=(n+1)-n / p$, then we have $\varepsilon>0$. Therefore, we have proved that the function $m=L^{i \gamma}(a)$ is a multiple of a $w$ - $(p, M, \varepsilon ; L)$-molecule. This completes the proof of Theorem 2 .

\section{Proof of Theorems 3 and 4}

Proof of Theorem 3. As in the proof of Theorem 1, assuming first that $f \in H_{L}^{p}(w) \cap L^{2}\left(\mathbb{R}^{n}\right)$, we are going to prove that, for every $w$ - $(p, M ; L)$-atom $a$ associated with a ball $B=B\left(x_{0}, r_{B}\right)$, $M>(3 n) / 4$, there exists a constant $C>0$ independent of $a$ such that $\left\|L^{-\alpha / 2}(a)\right\|_{L^{q}\left(w^{q / p}\right)} \leq C$. We write

$$
\begin{aligned}
& \left\|L^{-\alpha / 2}(a)\right\|_{L^{q}\left(w^{q / p}\right)}^{q} \\
& =\int_{2 B}\left|L^{-\alpha / 2}(a)(x)\right|^{q} w(x)^{q / p} d x \\
& \quad+\sum_{k=1}^{\infty} \int_{2^{k+1} B \backslash 2^{k} B}\left|L^{-\alpha / 2}(a)(x)\right|^{q} w(x)^{q / p} d x \\
& :=J_{1}+J_{2} .
\end{aligned}
$$

$$
\text { when } x \in 2^{j+1} B \backslash 2^{j} B \text {. }
$$


By our assumption $0<\alpha<n / 2,1 / q=1 / p-\alpha / n$, then we are able to choose a number $\mu>q$ such that $1 / \mu=1 / 2-\alpha / n$. Set $s=2 / p$. By a simple calculation, we can see that

$$
\begin{aligned}
\left(\frac{q}{p}\right) \cdot\left(\frac{\mu}{q}\right)^{\prime} & =\left(\frac{1}{p}\right) \cdot\left(\frac{q \mu}{(\mu-q)}\right) \\
& =\left(\frac{1}{p}\right) \cdot\left(\frac{1}{p}-\frac{1}{2}\right)^{-1}=s^{\prime}, \\
1-\frac{q}{\mu} & =q\left(\frac{1}{q}-\frac{1}{\mu}\right)=q\left(\frac{1}{p}-\frac{1}{2}\right)=\frac{q}{\left(p s^{\prime}\right)} .
\end{aligned}
$$

Applying Hölder's inequality with exponent $\nu=\mu / q>1$, the $L^{2}-L^{\mu}$ boundedness of $L^{-\alpha / 2}$ (see (10)), Lemma 5, and $w \in$ $\mathrm{RH}_{s^{\prime}}$, we get

$$
\begin{aligned}
J_{1} & \leq\left(\int_{2 B}\left|L^{-\alpha / 2}(a)(x)\right|^{q \cdot(\mu / q)} d x\right)^{q / \mu} \\
& \cdot\left(\int_{2 B} w(x)^{(q / p) \cdot(\mu / q)^{\prime}} d x\right)^{1-q / \mu} \\
& =\left(\int_{2 B}\left|L^{-\alpha / 2}(a)(x)\right|^{\mu} d x\right)^{q / \mu} \\
& \cdot\left(\int_{2 B} w(x)^{s^{\prime}} d x\right)^{q /\left(p s^{\prime}\right)} \leq C\|a\|_{L^{2}(B)}^{q}\left(\frac{w(2 B)}{|2 B|^{1 / s}}\right)^{q / p} \\
& \leq C .
\end{aligned}
$$

We now turn to deal with $J_{2}$. For any $x \in 2^{k+1} B \backslash 2^{k} B, k=$ $1,2, \ldots$, by expression (8), we can write

$$
\begin{aligned}
\left|L^{-\alpha / 2}(a)(x)\right| \leq & C \int_{0}^{\infty}\left|e^{-t L}(a)(x)\right| \frac{d t}{t^{1-\alpha / 2}} \\
\leq & C \int_{0}^{r_{B}^{2}}\left|e^{-t L}(a)(x)\right| \frac{d t}{t^{1-\alpha / 2}} \\
& +C \int_{r_{B}^{2}}^{\infty}\left|e^{-t L}(a)(x)\right| \frac{d t}{t^{1-\alpha / 2}} \\
:= & \text { III }+ \text { IV. }
\end{aligned}
$$

For the term III, it follows immediately from (54) that

$$
\begin{aligned}
\mathrm{III} & \leq C \cdot \frac{1}{2^{k(n+1)}[w(B)]^{1 / p}} \cdot \frac{1}{r_{B}} \int_{0}^{r_{B}^{2}} \frac{d t}{t^{1 / 2-\alpha / 2}} \\
& \leq C \cdot \frac{r_{B}^{\alpha}}{2^{k(n+1)}[w(B)]^{1 / p}} .
\end{aligned}
$$

For the other term IV, by the previous estimate (56), we thus have

$$
\begin{aligned}
\mathrm{IV} & \leq C \cdot \frac{1}{2^{k(n+1)}[w(B)]^{1 / p}} \cdot r_{B}^{2 M-1} \int_{r_{B}^{2}}^{\infty} \frac{d t}{t^{M+1 / 2-\alpha / 2}} \\
& \leq C \cdot \frac{r_{B}^{\alpha}}{2^{k(n+1)}[w(B)]^{1 / p}},
\end{aligned}
$$

where the last inequality holds since $M>(3 n) / 4>1 / 2+\alpha / 2$. Combining the above estimates for III and IV, we obtain the following pointwise inequality:

$$
\begin{aligned}
&\left|L^{-\alpha / 2}(a)(x)\right| \leq C \cdot \frac{r_{B}^{\alpha}}{2^{k(n+1)}[w(B)]^{1 / p}} \\
& \text { when } x \in 2^{k+1} B \backslash 2^{k} B .
\end{aligned}
$$

Note that $w \in A_{1}$. Substituting inequality (75) into the term $J_{2}$, we get

$$
J_{2} \leq C \sum_{k=1}^{\infty}\left(\frac{r_{B}^{\alpha}}{2^{k(n+1)}[w(B)]^{1 / p}}\right)^{q} \int_{2^{k+1} B} w(x)^{q / p} d x
$$

Using Hölder's inequality with exponent $v=\mu / q>1$, Lemma 5, and the condition $w \in A_{1} \cap \mathrm{RH}_{s^{\prime}}$, we can deduce that

$$
\begin{aligned}
J_{2} & \leq C \sum_{k=1}^{\infty}\left(\frac{r_{B}^{\alpha}}{2^{k(n+1)}[w(B)]^{1 / p}}\right)^{q} \cdot\left|2^{k+1} B\right|^{q / \mu} \\
& \cdot\left(\int_{2^{k+1} B} w(x)^{(q / p) \cdot(\mu / q)^{\prime}} d x\right)^{1-q / \mu} \\
& \leq C \sum_{k=1}^{\infty}\left(\frac{r_{B}^{\alpha}}{2^{k(n+1)}[w(B)]^{1 / p}}\right)^{q} \cdot\left|2^{k+1} B\right|^{q / \mu} \\
& \cdot\left(\frac{w\left(2^{k+1} B\right)}{\left|2^{k+1} B\right|^{1 / s}}\right)^{q / p} \leq C \sum_{k=1}^{\infty} \frac{1}{2^{k[q(n+1)-n]}} \leq C,
\end{aligned}
$$

where in the last inequality we have used the fact that $q>$ $p>n /(n+1)$. By combining inequality (77) with (71), we obtain the desired result. Therefore, by a standard density argument, we can show that the same conclusion holds for all $f \in H_{L}^{p}(w)$. This concludes the proof of Theorem 3 .

Proof of Theorem 4. As in the proof of Theorem 2, it is enough to show that, for every $w-(p, 2 M ; L)$-atom $a$, function $m=$ $L^{-\alpha / 2}(a)$ is a multiple of a $w-(p, M, \varepsilon ; L)$-molecule for some $\varepsilon>0$ and the multiplicative constant is independent of $a$. Let $a$ be a $w$ - $(p, 2 M ; L)$-atom with supp $a \subseteq B=B\left(x_{0}, r_{B}\right)$ and $a=L^{2 M}(b)$, where $M>(3 n) / 4>\max \{(n / 2)(1 / p-1 / 2), 1 / 2+$ $\alpha / 2\}, b \in \mathscr{D}\left(L^{2 M}\right)$. Setting $\widetilde{b}=L^{-\alpha / 2}\left(L^{M} b\right)$, then we have $m=L^{M}(\tilde{b})$. It is easy to check that $m(x) \in L^{2}\left(\mathbb{R}^{n}\right)$. As before, since $0<\alpha<n / 2$, then we may choose a number $\mu>2$ such that $1 / \mu=1 / 2-\alpha / n$. For $k=0,1, \ldots, M$, by using Hölder's inequality and the $L^{2}-L^{\mu}$ boundedness of $L^{-\alpha / 2}$, we obtain

$$
\begin{aligned}
& \left\|\left(r_{B}^{2} L\right)^{k} \tilde{b}\right\|_{L^{2}(2 B)} \\
& \leq \frac{1}{r_{B}^{2 M}}\left\|L^{-\alpha / 2}\left[\left(r_{B}^{2} L\right)^{M+k} b\right]\right\|_{L^{\mu}(2 B)}|2 B|^{1 / 2-1 / \mu} \\
& \leq C \cdot \frac{1}{r_{B}^{2 M}}\left\|\left(r_{B}^{2} L\right)^{M+k} b\right\|_{L^{2}(B)}|B|^{1 / 2-1 / \mu} \\
& \leq C \cdot r_{B}^{2 M}|B|^{1 / 2+\alpha / n}[w(B)]^{-1 / p} .
\end{aligned}
$$


Noting that $1 / q=1 / p-\alpha / n$, then a straightforward computation yields that $q / p<(2 / p)^{\prime}$ whenever $0<\alpha<n / 2$. By our assumption $w \in \mathrm{RH}_{(2 / p)^{\prime}}$, then we have $w \in \mathrm{RH}_{q / p}$. Consequently,

$$
\left[w^{q / p}(B)\right]^{p / q} \leq C \cdot \frac{w(B)}{|B|^{1-p / q}}
$$

which implies

$$
[w(B)]^{-1 / p} \leq C \cdot|B|^{1 / q-1 / p}\left[w^{q / p}(B)\right]^{-1 / q} .
$$

Substituting inequality (80) into (78), we can get

$$
\left\|\left(r_{B}^{2} L\right)^{k} \tilde{b}\right\|_{L^{2}(2 B)} \leq C \cdot r_{B}^{2 M}|B|^{1 / 2}\left[w^{q / p}(B)\right]^{-1 / q} .
$$

It remains to estimate $\left\|\left(r_{B}^{2} L\right)^{k} \widetilde{b}\right\|_{L^{2}\left(2^{j+1} B \backslash 2^{j} B\right)}$ for $k=0,1, \ldots$, $M, j=1,2, \ldots$. We write

$$
\begin{aligned}
\left|\left(r_{B}^{2} L\right)^{k} \widetilde{b}(x)\right|= & \left|L^{-\alpha / 2}\left[\left(r_{B}^{2} L\right)^{k} L^{M} b\right](x)\right| \\
\leq & C \int_{0}^{r_{B}^{2}}\left|e^{-t L}\left[r_{B}^{2 k} L^{M+k} b\right](x)\right| \frac{d t}{t^{1-\alpha / 2}} \\
& +C \int_{r_{B}^{2}}^{\infty}\left|e^{-t L}\left[r_{B}^{2 k} L^{M+k} b\right](x)\right| \frac{d t}{t^{1-\alpha / 2}} \\
:= & \mathrm{III}^{\prime}+\mathrm{IV}^{\prime} .
\end{aligned}
$$

For the term III', by using the same arguments as in the proof of (63), we have

$$
\begin{aligned}
\mathrm{III}^{\prime} & \leq C \cdot \frac{1}{2^{j(n+1)}} \cdot r_{B}^{2 M}[w(B)]^{-1 / p} \frac{1}{r_{B}} \int_{0}^{r_{B}^{2}} \frac{d t}{t^{1 / 2-\alpha / 2}} \\
& \leq C \cdot \frac{1}{2^{j(n+1)}} \cdot r_{B}^{2 M+\alpha}[w(B)]^{-1 / p} .
\end{aligned}
$$

For the term $I^{\prime}$, we proceed as that of (66) and then obtain

$$
\begin{aligned}
\mathrm{IV}^{\prime} \leq & C \cdot \frac{1}{2^{j(n+1)}} \\
& \cdot[w(B)]^{-1 / p} r_{B}^{4 M+2 k-1} \int_{r_{B}^{2}}^{\infty} \frac{d t}{t^{M+k+1 / 2-\alpha / 2}} \\
\leq & C \cdot \frac{1}{2^{j(n+1)}} \cdot r_{B}^{2 M+\alpha}[w(B)]^{-1 / p} .
\end{aligned}
$$

Combining the above estimates for III' and $\mathrm{IV}^{\prime}$, we can get

$$
\begin{array}{r}
\left|\left(r_{B}^{2} L\right)^{k} \widetilde{b}(x)\right| \leq C \cdot \frac{1}{2^{j(n+1)}} \cdot r_{B}^{2 M+\alpha}[w(B)]^{-1 / p}, \\
\text { when } x \in 2^{j+1} B \backslash 2^{j} B .
\end{array}
$$

Since $w \in A_{1}$, then it follows from inequality (64) that

$$
\begin{aligned}
\left|\left(r_{B}^{2} L\right)^{k} \tilde{b}(x)\right| \leq & C \cdot \frac{1}{2^{j[(n+1)-n / p]}} \\
& \cdot r_{B}^{2 M+\alpha}\left[w\left(2^{j} B\right)\right]^{-1 / p},
\end{aligned}
$$

when $x \in 2^{j+1} B \backslash 2^{j} B$.
Similar to the proof of (80), we can also show that

$$
\left[w\left(2^{j} B\right)\right]^{-1 / p} \leq C \cdot\left|2^{j} B\right|^{1 / q-1 / p}\left[w^{q / p}\left(2^{j} B\right)\right]^{-1 / q} .
$$

Hence, we finally obtain

$$
\begin{aligned}
\left|\left(r_{B}^{2} L\right)^{k} \tilde{b}(x)\right| \leq & C \cdot \frac{1}{2^{j[(n+1)-n / q]}} \\
& \cdot r_{B}^{2 M}\left[w^{q / p}\left(2^{j} B\right)\right]^{-1 / q},
\end{aligned}
$$

when $x \in 2^{j+1} B \backslash 2^{j} B$.

Therefore,

$$
\begin{aligned}
& \left\|\left(r_{B}^{2} L\right)^{k} \tilde{b}\right\|_{L^{2}\left(2^{j+1} B \backslash 2^{j} B\right)} \\
& \quad \leq C \cdot \frac{1}{2^{j[(n+1)-n / q]}} \cdot r_{B}^{2 M}\left|2^{j} B\right|^{1 / 2}\left[w^{q / p}\left(2^{j} B\right)\right]^{-1 / q} .
\end{aligned}
$$

Observe that $1 \geq q>p>n /(n+1)$. If we set $\varepsilon=(n+1)-$ $n / q$, then $\varepsilon>0$. Summarizing estimates (81) and (89) derived above, we conclude the proof of Theorem 4 .

\section{Conflict of Interests}

The author declares that there is no conflict of interests regarding the publication of this paper.

\section{References}

[1] B. Simon, "Maximal and minimal Schrödinger forms," Journal of Operator Theory, vol. 1, no. 1, pp. 37-47, 1979.

[2] Z. W. Shen, " $L^{p}$ estimates for Schrodinger operators with certain potentials," Annales de l'Institut Fourier, vol. 45, pp. 513-546, 1995.

[3] H. Gunawan, "Some weighted estimates for imaginary powers of Laplace operators," Bulletin of the Australian Mathematical Society, vol. 65, no. 1, pp. 129-135, 2002.

[4] A. Sikora and J. Wright, "Imaginary powers of Laplace operators," Proceedings of the American Mathematical Society, vol. 129, no. 6, pp. 1745-1754, 2001.

[5] E. M. Stein, Singular Integrals and Differentiability Properties of Functions, Princeton University Press, Princeton, NJ, USA, 1970.

[6] P. Auscher and J. M. Martell, "Weighted norm inequalities for fractional operators," Indiana University Mathematics Journal, vol. 57, no. 4, pp. 1845-1870, 2008.

[7] X. T. Duong and L. X. Yan, "On commutators of fractional integrals," Proceedings of the American Mathematical Society, vol. 132, no. 12, pp. 3549-3557, 2004.

[8] H. X. Mo and S. Z. Lu, "Boundedness of multilinear commutators of generalized fractional integrals," Mathematische Nachrichten, vol. 281, no. 9, pp. 1328-1340, 2008.

[9] L. X. Yan, "Classes of Hardy spaces associated with operators, duality theorem and applications," Transactions of the American Mathematical Society, vol. 360, no. 8, pp. 4383-4408, 2008.

[10] L. Song and L. X. Yan, "Riesz transforms associated to Schrödinger operators on weighted Hardy spaces," Journal of Functional Analysis, vol. 259, no. 6, pp. 1466-1490, 2010. 
[11] J. Garcia-Cuerva, "Weighted $H^{p}$ spaces," Dissertations Math, vol. 162, pp. 1-63, 1979.

[12] J.-O. Stömberg and A. Torchinsky, Weighted Hardy spaces, vol. 1381 of Lecture Notes in Mathematics, Springer-Verlag, 1989.

[13] H. Wang, "Riesz transforms associated with Schrodinger operators acting on weighted Hardy spaces," Analysis in Theory and Applications, vol. 31, no. 2, pp. 138-153, 2015.

[14] B. Muckenhoupt, "Weighted norm inequalities for the Hardy maximal function," Transactions of the American Mathematical Society, vol. 165, pp. 207-226, 1972.

[15] J. Garcia-Cuerva and J. Rubio de Francia, Weighted Norm Inequalities and Related Topics, North-Holland, Amsterdam, The Netherlands, 1985.

[16] E. B. Davies, Heat Kernels and Spectral Theory, Cambridge University Press, Cambridge, 1989.

[17] E. M. Ouhabaz, Analysis of Heat Equations on Domains, vol. 31 of London Mathematical Society Monographs, Princeton University Press, Princeton, NJ, USA, 2005.

[18] P. Auscher, X. T. Duong, and A. McIntosh, "Boundedness of Banach space valued singular integral operators and Hardy spaces," Preprint, 2005.

[19] S. Hofmann, G. Z. Lu, D. Mitrea, M. Mitrea, and L. X. Yan, "Hardy spaces associated to non-negative self-adjoint operators satisfying Davies-Gaffney estimates," Memoirs of the American Mathematical Society, vol. 214, no. 1007, 2011.

[20] A. Sikora, "Riesz transform, Gaussian bounds and the method of wave equation," Mathematische Zeitschrift, vol. 247, no. 3, pp. 643-662, 2004.

[21] X. T. Duong and L. X. Yan, "Spectral multipliers for Hardy spaces associated to non-negative self-adjoint operators satisfying Davies-Gaffney estimates," Journal of the Mathematical Society of Japan, vol. 63, no. 1, pp. 295-319, 2011.

[22] S. Hofmann and S. Mayboroda, "Hardy and BMO spaces associated to divergence form elliptic operators," Mathematische Annalen, vol. 344, no. 1, pp. 37-116, 2009.

[23] D. G. Deng, X. T. Duong, A. Sikora, and L. X. Yan, “Comparison of the classical BMO with the BMO spaces associated with operators and applications," Revista Matemática Iberoamericana, vol. 24, no. 1, pp. 267-296, 2008. 


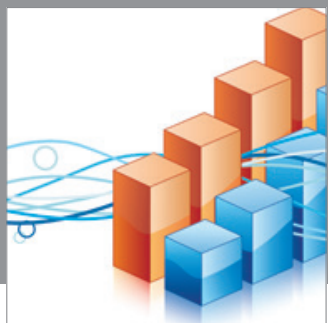

Advances in

Operations Research

mansans

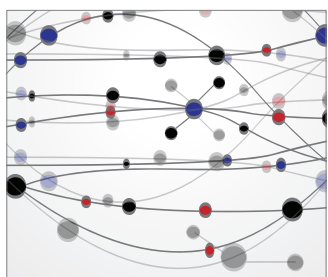

The Scientific World Journal
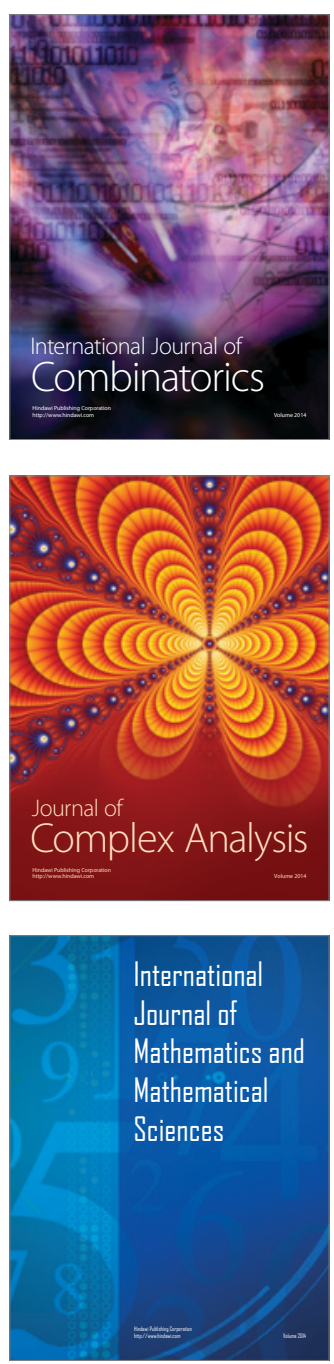
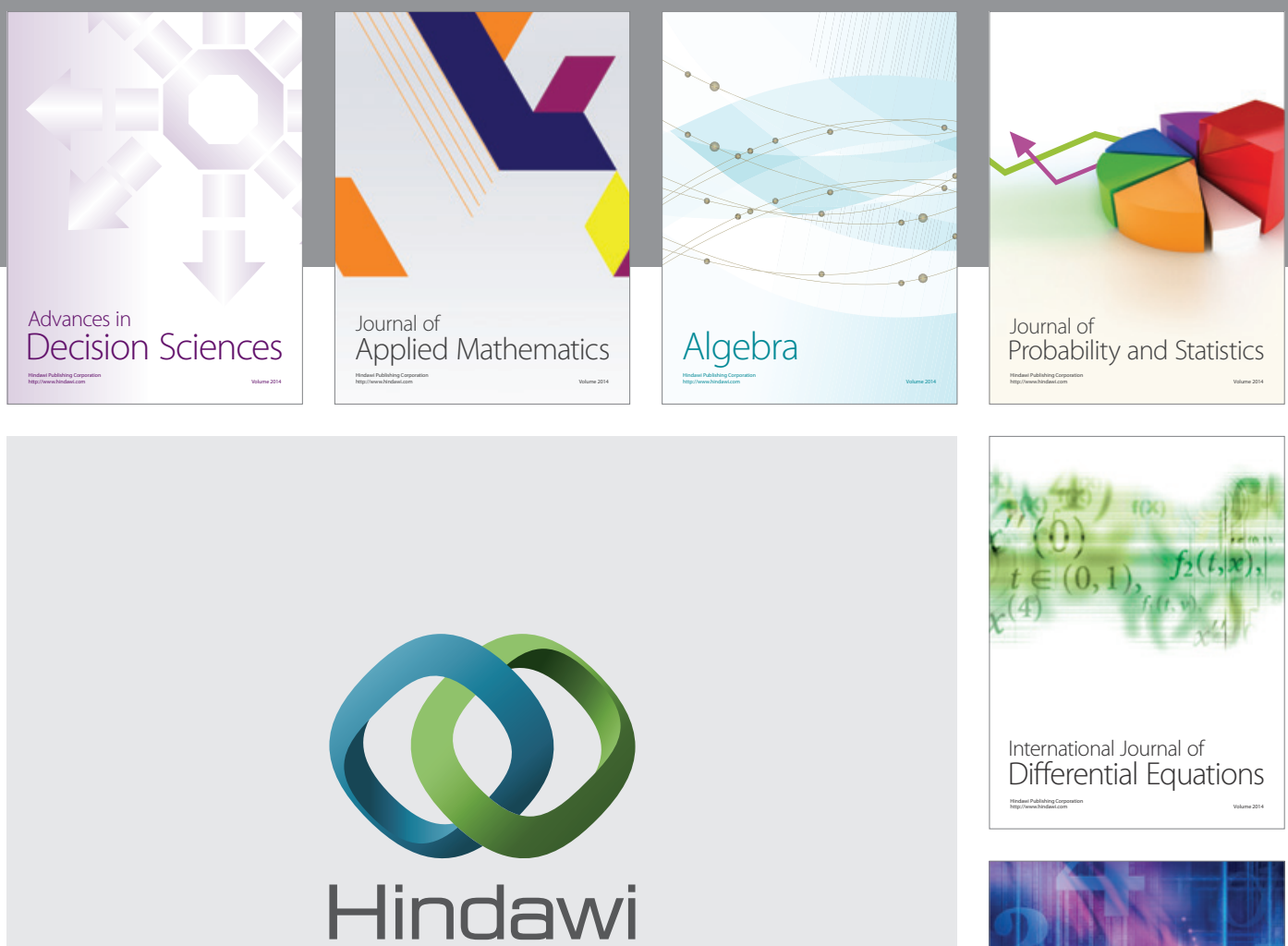

Submit your manuscripts at http://www.hindawi.com
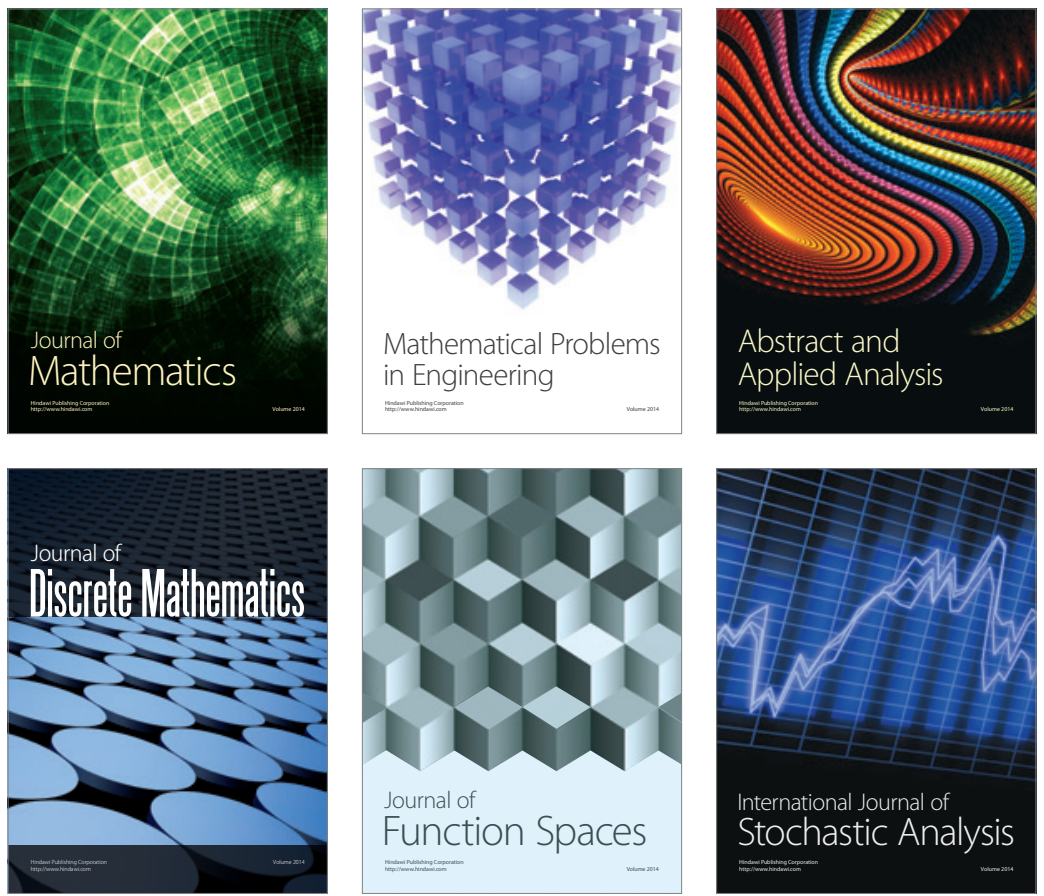

Journal of

Function Spaces

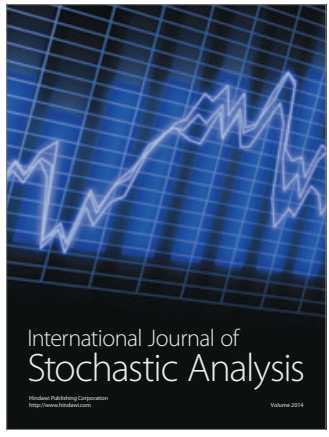

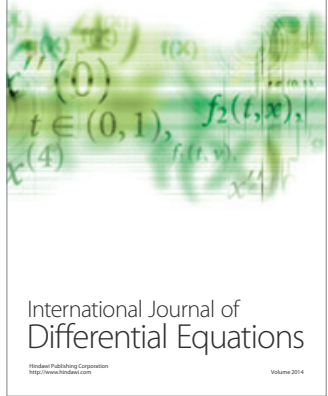
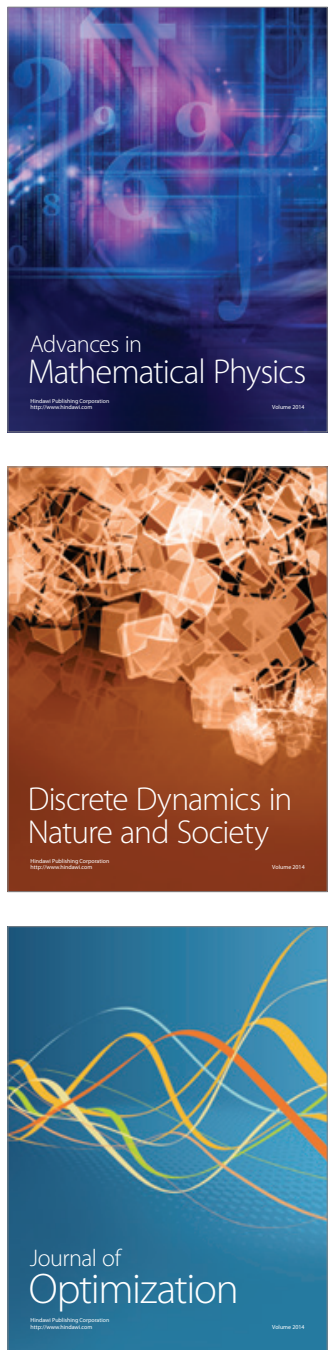